\title{
Improving electrochemical biosensor performance by understanding the influence of target DNA length on assay sensitivity.
}

Damion K Corrigan ${ }^{1}$, Holger Schulze ${ }^{2}$, Rachel A McDermott ${ }^{1}$, Ilka Schmüser ${ }^{1 \& 3}$, Grace Henihan ${ }^{2}$, John B Henry ${ }^{1}$, Till T Bachmann ${ }^{2}$, Andrew R Mount ${ }^{1}$.

${ }^{1}$ EaStCHEM, School of Chemistry, The University of Edinburgh, Joseph Black Building, The King's Buildings, West Mains Road, Edinburgh, EH9 3JJ, Scotland (UK

2 Division of Pathway Medicine, School of Biomedical Sciences, The University of Edinburgh, Chancellor's Building, Little France Crescent, Edinburgh EH16 4SB, Scotland (UK)

3 Institute for Integrated Micro and Nano Systems, School of Engineering, The University of Edinburgh, The King's Buildings, West Mains Road, Edinburgh EH9 3JF, Scotland (UK)

Corresponding author - Dr Damion Corrigan, School of Chemistry, The University of Edinburgh, Joseph Black Building, The King's Buildings, West Mains Road, Edinburgh, EH9 3JJ, Scotland (UK). Email - damioncorrigan@ed.ac.uk

\begin{abstract}
Electrochemical DNA hybridisation assays allow the measurement of probe-target binding in a label free fashion for biosensor and healthcare detection, particularly when using electrochemical impedance spectroscopy (EIS). Typically in EIS assay development a short artificial oligonucleotide is used to test sensor performance and consideration is not given to binding real world samples which can contain DNA fragments of varying length. This paper investigates the effect of solution side DNA overhangs on the EIS signal. Firstly, by using a surface tethered 15 nucleotides (nt) PNA probe and a series of artificial DNA targets with increasingly long overhangs it was possible to measure differences in the EIS signal brought about by target length. It was found that an overhang of $15 \mathrm{nt}$ gave the most effective enhancement of signal. This enhancement was attributed to the overhang reducing the access of the redox couple to the electrode surface. Overhangs of 45 and $85 \mathrm{nt}$ caused a smaller enhancement of signal which can be attributed to the reduced hybridisation efficiency of longer strands. Secondly, EIS responses from fragmented Methicillin resistant Staphylococcus aureus (MRSA) bacterial genomic DNA (gDNA) were measured and it was found that in the case of the MRSA gDNA, progressive fragmentation of long DNA sequences (>1000 bp) to less than $100 \mathrm{bp}$ coincided with enhancement of the EIS signal. From the results obtained with oligonucleotides it is inferred that the creation of shorter solution side overhangs in real world MRSA samples boosts the electrochemical signal observed upon DNA binding.
\end{abstract}

\section{Introduction}

Electrochemical impedance spectroscopy (EIS) can be used to measure biological binding at an electrode surface ${ }^{1}$. It is an attractive technology because it allows label free measurement of DNA hybridisation and electrochemical sensors are easily and cheaply produced ${ }^{2}$. This approach typically uses a three electrode system; the dc potential of the working electrode is held at a value to fix the 
local redox composition and upon which a small sinusoidal ac signal is superimposed. The resulting current response of the cell is measured, from which the physical parameters associated with the cell such as the solution resistance $\left(\mathrm{R}_{\mathrm{S}}\right)$, double layer capacitance $\left(\mathrm{C}_{\mathrm{DL}}\right)$ charge transfer resistance $\left(\mathrm{R}_{\mathrm{CT}}\right)$ and the Warburg Impedance $\left(Z_{W}\right)$ are obtained by fitting to the Randles' equivalent circuit ${ }^{3}$. In the monitoring of nucleic acid binding, EIS experiments usually involve the use of a negatively charged redox couple such as potassium ferri/ferrocyanide and monitoring changes in $\mathrm{R}_{\mathrm{CT}}$ brought about by DNA hybridisation. A large number of studies have been published which show the development of EIS based DNA biosensors ${ }^{4-13}$. For example, the development of sensitive assays for protein and DNA binding have been reported ${ }^{14 ; 15}$. One approach to enhancing the detection of DNA hybridisation by EIS is to use a neutral PNA ${ }^{7 ; 15-17}$ or morpholino probe ${ }^{18}$. This is because it is considered that a neutrally charged probe molecule allows improved resolution of binding when the negatively charged DNA target strands hybridise to the probe molecule at the electrode surface through the combination of enhanced repulsion and steric hindrance of the negatively charged solution redox probe typically employed. Work with morpholino probes has been shown to lead to detection using capacitive measurements where no redox agent is solution ${ }^{18}$ and detection levels of pictograms $/ \mathrm{cm}^{2}$ were shown as possible. In previous studies we employed a redox mediator based strategy to develop a sensitive test for PCR generated fragments of MRSA DNA $(10 \mathrm{pM}){ }^{15}$ and fragments of chromosomal MRSA gDNA (50 fM) ${ }^{3}$. In these studies it was noted that target length had an effect on EIS signal strength and assay variation. Studies of target length effect on EIS signal have been reported for DNA-DNA interactions ${ }^{19}$ but as yet have not been reported for PNA-DNA. In this paper the effect of using artificial oligonucleotide sequences with controlled length variations is studied and related to new findings from clinical samples with the aim of understanding this enhanced biosensor performance.

Specifically, a 15 base pair PNA probe was employed to bind a series of four fully complementary DNA oligonucleotides. The oligonucleotides varied in length on the solution side of the probe sequence. The lengths of the four oligonucleotides were 15, 30, 60 and $100 \mathrm{nt}$ giving solution side overhangs of $0,15,45$ and $85 \mathrm{nt}$. To evaluate the effect in real world samples, experiments were then performed using samples of fragmented MRSA gDNA. The patterns observed in the binding of the artificial targets and bacterial DNA were compared in order to gain insight into the effects of target length on sensitivity.

\section{Materials and Methods}

Potasssium ferri and ferrocyanide, sodium mono and diphosphate buffer and oligonucleotides were purchased from Sigma Aldrich (Poole, Dorset, UK). The thiolated PNA probe sequences were obtained from Panagene (Daejeon, South Korea). EIS measurements were performed using a three electrode cell consisting of a $1.6 \mathrm{~mm}$ diameter gold macrodisc working electrode (IJ Cambria, Llanelli, UK), $\mathrm{Ag} / \mathrm{AgCl} / \mathrm{Cl}^{-}(3 \mathrm{M})$ reference electrode Metrohm (Windsor, UK) and a platinum gauze counter electrode under potentiostatic control (Metrohm, Windsor, UK). A frequency range of 100 $\mathrm{kHz}$ to $0.1 \mathrm{~Hz}$ was employed with signal amplitude of $10 \mathrm{mV}$ rms at the measured open circuit potential (which with equal concentrations of ferri and ferrocyanide in the solution was the formal potential of the potassium ferri-ferrocyanide couple). 25 data points were collected in order to ensure measurement times of less than 1.5 minutes (as they would need to be for a real world sensor). EIS experiments were performed pre and post hybridisation with a one hour incubation of the electrode and target DNA - this was called "Batch Mode". Protocols for electrode cleaning, functionalisation, electrochemical measurements and bacterial DNA extraction can be found in previous studies ${ }^{3 ; 14}$. Samples of MRSA gDNA were incubated at $95^{\circ} \mathrm{C}$ in $2 \mathrm{xSSC}$ for $0,1,5$ and 10 minutes in order to achieve progressive fragmentation. Fragmented samples were subjected to DNA gel electrophoresis using an Agilent Bioanalyzer 4100 system (Agilent, Wokingham, UK). 
Table 1 - DNA and oligonucleotide sequences used in this study

\begin{tabular}{|c|c|c|c|c|c|}
\hline & $\begin{array}{l}\text { Oligo } \\
\text { name }\end{array}$ & Type & $\begin{array}{c}3^{\prime} \\
\text { Modif. }\end{array}$ & $\begin{array}{c}5^{\prime} \\
\text { Modif. }\end{array}$ & Sequence 5'-3' \\
\hline $\mathrm{T} 1$ & 15 mer & DNA & - & - & TCTGCATTCCTGGAA \\
\hline $\mathrm{T} 2$ & 30 mer & DNA & - & - & GTATGCTTTGGTCTTTCTGCATTCCTGGAA \\
\hline $\mathrm{T} 3$ & 60 mer & DNA & - & - & $\begin{array}{c}\text { TACCACGTTCTGATTTTAAATTTTC } \\
\text { AATATGTATGCTTTGGTCTTTCTGCATTCCTGGAA }\end{array}$ \\
\hline $\mathrm{T} 4$ & $\begin{array}{l}100 \\
\text { mer }\end{array}$ & DNA & - & - & $\begin{array}{c}\text { CCTGTATTGGCCAATTCCACATTGTTT } \\
\text { CGGTCTAAAATTTTACCACGTTCTGATTTTAAATTTTCAA } \\
\text { TATGTATGCTTTGGTCTTTCTGCATTCCTGGAA }\end{array}$ \\
\hline $\mathrm{P} 1$ & $\begin{array}{c}\text { PNA } \\
\text { MRSA }\end{array}$ & PNA & - & $\begin{array}{c}3.8 \mathrm{~nm}- \\
\text { Thiol- } \\
\text { C11- } \\
\text { AEEEA }\end{array}$ & TTCCAGGAATGCAGA \\
\hline P2 & $\begin{array}{l}\text { PNA } \\
48 \_02\end{array}$ & PNA & - & $\begin{array}{c}3.8 \mathrm{~nm}- \\
\text { Thiol- } \\
\text { C11- } \\
\text { AEEEA }\end{array}$ & ACTAGGTGTTGGTGAAGATATAC \\
\hline & & & & & $\begin{array}{c}\mathrm{T}=\text { target } \\
\mathrm{P}=\text { probe sequence } \\
\underline{\text { Bold }}=\text { probe binding regions within target DNA sequences }\end{array}$ \\
\hline
\end{tabular}

Prior to film immobilisation the electrodes were cleaned by dipping in piranha solution (caution: take care when handling this solution) for 10 minutes followed by cyclic voltammetry in $0.1 \mathrm{M}$ sulfuric acid. Immobilisation was carried out by exposing the electrode to a $1.5 \mu \mathrm{M}$ PNA probe and $30 \mu \mathrm{M} 6$ mercapto-1-hexanol solution for 16 hours followed by blocking in $1 \mathrm{mM}$ 6-mercapto-1-hexanol solution for 1 hour. An increase in $\mathrm{R}_{\mathrm{CT}}$ was noted following incubation of the electrode with $100 \mathrm{nM}$ target DNA in $100 \mathrm{mM} \mathrm{KCl}$ solution for 1 hour. Figure 1A provides an illustration of the change in the Nyquist plot upon DNA binding at the electrode surface. Such increases were expressed by dividing $\mathrm{R}_{\text {Ст }}$ post hybridisation by $\mathrm{R}_{\mathrm{CT}}$ prehybridisation to obtain the "Signal Ratio". The increases observed were consistent with previous studies where appropriate control experiments were performed to ensure signal increases were in fact due to DNA hybridisation ${ }^{15}$. Data were fitted using the Randles' equivalent circuit and Autolab FRA software (Figure 1B) and errors for $\mathrm{R}_{\mathrm{CT}}$ were in the range of $6-10 \%$ for these fits and this was consistent with previous studies.

\section{Results and Discussion}

The test was initially run by continuously performing EIS measurements and adding DNA directly into the EIS measurement buffer $(10 \mathrm{mM} \mathrm{pH} 7.0 \text { phosphate buffer }+0.1 \mathrm{mM} \text { ferri/ferrocyanide })^{17}$, see Figure 1C. This format was called "kinetic mode" and it was seen that the 30 mer caused the largest increase in EIS signal. In this case of continuous monitoring of binding in EIS measurement buffer, the 15 mer gave the second largest increase in signal followed by the 60 mer and finally the 100 mer. Whilst the recognition sequence stayed the same, the increasing overhang length made target binding thermodynamically less favourable. It can be seen from Figure $1 \mathrm{C}$ that the process was not kinetically 
limited and so for batch mode an incubation time of 1 hour was selected in order to ensure that all binding processes were at equilibrium.

To further test the effect of target length on EIS signal increases a simple batch end point assay was performed as described previously where EIS spectra were recorded pre and post hybridisation (Figure 1D). It can be seen that the 30 mer (15 nt overhang) again caused the largest increase in the EIS signal. The 60 mer gave the second largest signal ratio increase and the 15 and 100 mers each produced lower signal increases. All incubations were made using oligonucleotide solutions of $1 \mu \mathrm{M}$ concentrations in $100 \mathrm{mM} \mathrm{KCl}$ for 1 hour. Table 2 summarises the values for all circuit parameters pre and post hybridisation for an electrode incubated with the 30 mer (15nt overhang) for 1 hour. Table 3 displays the signal ratios for the various circuit parameters and demonstrates that changes in $\mathrm{R}_{\mathrm{CT}}$ gave the most reliable indication of DNA hybridisation due to this being the parameter associated with the probe film and therefore the sensing of any changes in the film properties brought about by the insertion of DNA target strands. 

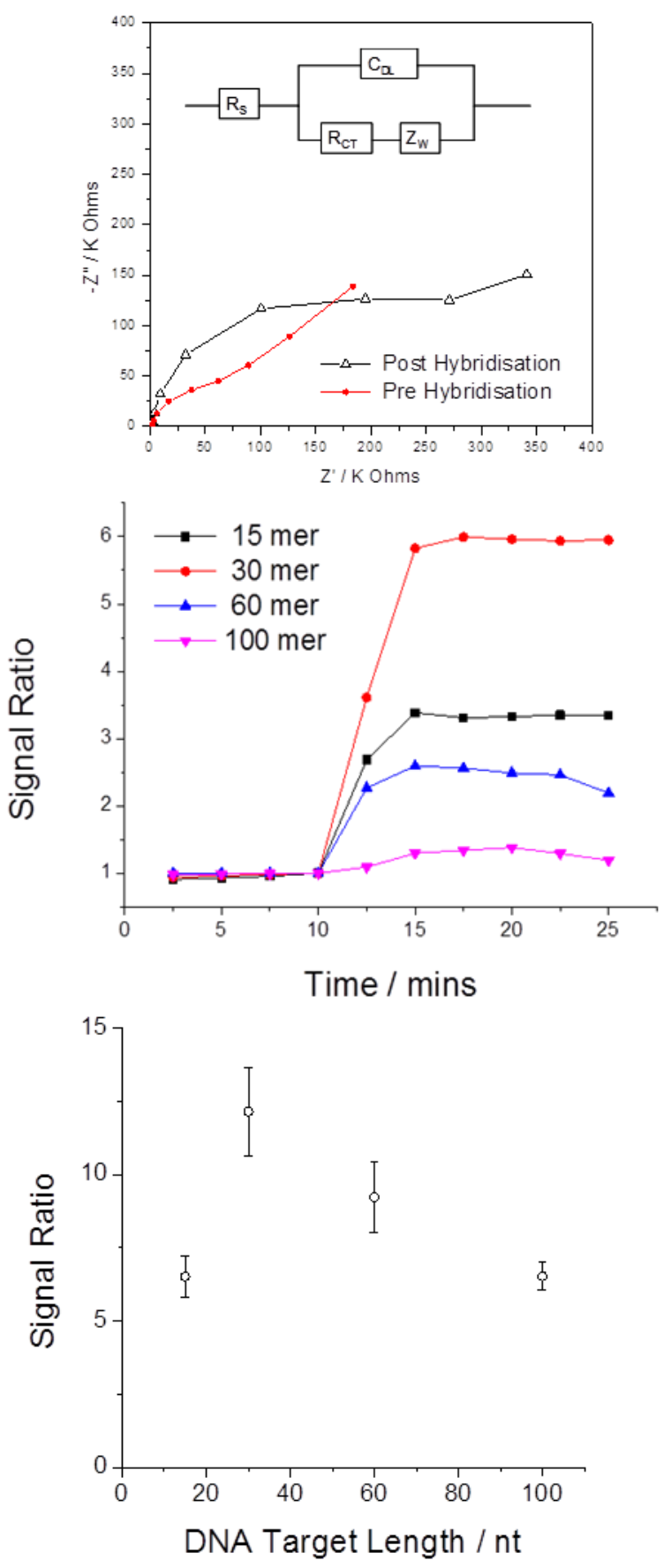

Figure 1. (A) Nyquist plot showing EIS response pre and post hybridisation with $100 \mathrm{nM}$ target DNA for a probe 1 functionalised electrode to illustrate the observed increase in the charge transfer semi-circle. (B) Randles' equivalent circuit. Including the circuit elements $\mathbf{R}_{\mathbf{S}}-$ solution resistance, $C_{D L}$ - double layer capacitance, $R_{C T}-$ charge transfer resistance and $Z_{W}-$ Warburg impedance (C) Signal Increases following incubation with four DNA targets of varying length (D) Signal increases for all four DNA target lengths $(1 \mu \mathrm{M})$ at probe 1 modified electrodes following a period of baseline stabilisation.

Table 2. Circuit parameters pre and post hybridisation for a probe 1 modified electrode exposed to the $30 \mathrm{nt}$ target. 


\begin{tabular}{|c|c|c|c|c|c|}
\hline & $\mathbf{R}_{\mathbf{S}}(\mathbf{k} \boldsymbol{\Omega})$ & $\mathbf{C}_{\mathbf{D L}}(\mathbf{n F})$ & $\mathbf{R}_{\mathbf{C T}}(\mathbf{k} \boldsymbol{\Omega})$ & $\mathbf{Q}(\boldsymbol{\mu} \mathbf{F})$ & $\mathbf{n}$ \\
\hline Pre & 1.98 & 306.56 & 37.84 & 7.53 & 0.45 \\
\hline Error & $1.02 \%$ & $2.00 \%$ & $2.92 \%$ & $071 \%$ & $0.90 \%$ \\
\hline & & & & & \\
\hline Post & 1.94 & 360.90 & 448.71 & 3.35 & 0.48 \\
\hline Error & $2.19 \%$ & $0.84 \%$ & $1.75 \%$ & $6.18 \%$ & $6.36 \%$ \\
\hline
\end{tabular}

Table 3. Signal Ratio changes in batch mode for a single Probe 1 modified electrode after incubation with DNA targets of varying lengths.

\begin{tabular}{|c|c|c|c|c|}
\hline Signal Ratio & $\mathbf{1 5} \mathbf{n t}$ & $\mathbf{3 0} \mathbf{n t}$ & $\mathbf{6 0} \mathbf{n t}$ & $\mathbf{1 0 0} \mathbf{n t}$ \\
\hline $\mathbf{C}_{\mathrm{DL}}$ & 1.2 & 1.2 & 1.3 & 1.1 \\
\hline $\mathbf{R}_{\mathrm{CT}}$ & 6.3 & 14.3 & 10.6 & 9.8 \\
\hline $\mathrm{Z}_{\mathrm{W}}$ & 0.8 & 0.6 & 0.7 & 0.7 \\
\hline
\end{tabular}

The batch assay involved incubation of the electrode with target DNA in a $100 \mathrm{mM} \mathrm{KCl}$ solution. This approach has been previously employed to ensure adequate hybridisation of DNA on modified electrode surfaces ${ }^{20}$. Binding will be different in an EIS measurement buffer of low ionic strength such as those employed for continuous biological measurements with EIS ${ }^{17}$. For EIS it is necessary to use a measurement buffer of low ionic strength in order to resolve DNA binding at an electrode surface. Whilst longer fragments would create a more repulsive barrier they must overcome selfrepulsive forces when inserting into the probe film and this explains why there was a significant decrease in binding from longer fragments in kinetic mode where probe-target binding took place in a solution of low ionic strength. Figure 1D shows that in batch mode the 60 mer caused the second highest increase in EIS signal whereas Figure 1C shows that in kinetic mode the 15 mer produced the second highest signal increase. In the batch assay where probe-target hybridisation was allowed to take place in a solution of higher ionic strength $(100 \mathrm{mM} \mathrm{KCl})$, due to the reduced charge screening and decreased Donnan potential in the film, it was possible to more effectively overcome the repulsive forces between target strands and therefore insert comparatively more of the long DNA fragments into the sensing film. This explains the difference in the observed pattern of signal ratio increases between the kinetic and batch assays and also why in in all cases Signal Ratio increases were greatest in the batch assay.

$$
\delta=\frac{1}{\kappa}=\left[\frac{\varepsilon k T}{e^{2} \sum_{i} c_{i} z_{i}}\right]^{1 / 2}
$$

Equation 1 - the expression used to calculate the Debye length ${ }^{21}(\delta) . \kappa=\mathrm{cm}^{-1}, e=\operatorname{charge}^{2}$ of an electron, $\varepsilon=$ permittivity $\varepsilon_{\mathrm{R}} \varepsilon_{0} . k=$ the Boltzmann constant and $T=$ temperature in $\mathrm{K}$.

Assuming a rod like structure for the DNA, the length of the four target strands were 4.95, 9.9, 19.8 and $33.0 \mathrm{~nm}$ respectively. The Debye length was calculated as $3.1 \mathrm{~nm}$ in the EIS measurement buffer and $0.97 \mathrm{~nm}$ in $100 \mathrm{mM} \mathrm{KCl}$ using equation 1 . The 30 mer with a $15 \mathrm{nt}$ overhang produced the largest increases in Signal Ratio and this can be explained in terms of a trade-off between electrostatic repulsion of the redox couple and hybridisation efficiency. The extra $15 \mathrm{nts}$ overhanging the probe sequence into solution served to increase the repulsive barrier at the electrode surface but was short enough to not encounter appreciable difficulty inserting into the sensing film. In effect the additional 
15 nt served as a "label" which boosted the EIS signal when compared to a target strand of the same length as the probe sequence ( 15 mer). When compared to the 15 mer the longer 60 mer with a $45 \mathrm{nt}$ overhang was able to enhance the signal in batch mode when the higher ionic strength $100 \mathrm{mM} \mathrm{KCl}$ solution was used for hybridisation. Again this is due to the decrease in the Debye length and the ability to insert more target DNA strands into the film. We have previously reported non Langmuir like insertion into electrode immobilised sensing films ${ }^{14}$ due to effects from neighbouring probes. In this case the effect is due to electrostatic interactions rather than steric hindrance. The basic model is that two processes are at work, firstly, target strands must overcome self-repulsive forces in order to insert into the film and secondly, once inserted, overhanging sequence providing an enhancement to the assay signal through repulsion of redox mediator. It is now our intention to thoroughly measure the hybridisation kinetics $\left(\mathrm{k}_{\mathrm{on}}\right.$ and $\left.\mathrm{k}_{\mathrm{off}}\right)$ for the different artificial targets, assess the effect of probe surface coverage and perform more detailed DNA fragmentation experiments.

\section{Genomic DNA}

Clinical samples are complex in terms of interfering compounds and the integrity of the target DNA and by obtaining an understanding of the influence of target size on EIS signal it may be possible to further optimise electrochemical DNA hybridisation tests. It has been previously shown that it is possible to detect bacterial ssDNA by treating dsDNA at $95^{\circ} \mathrm{C}$ for $5 \mathrm{mins}{ }^{22}$ and that when done so in 2xSSC there is significant fragmentation of the long bacterial chromosomal DNA sequences ${ }^{15}$. MRSA is an antibiotic resistant bacterium of extreme clinical significance. With the increased prevalence of antibiotic resistance there is a pressing requirement for rapid and sensitive MRSA tests. In this work, MRSA gDNA extracted from suspensions of $10^{7} \mathrm{cells} / \mathrm{mL}$ was heat treated at $95^{\circ} \mathrm{C}$ over a range of times and the fragmentation pattern analysed by capillary gel electrophoresis (see Figure 2 A-D). Additionally, the EIS signal ratio brought about by batch mode incubation with the heat treated samples was measured. To perform the EIS measurements on MRSA gDNA a different PNA sequence (P2) was used because it has been shown to effectively bind clinical samples of MRSA gDNA $^{3}$. Figure 2 (A-D) shows that as the MRSA DNA fragmentation time increased the target DNA was found to be present in shorter fragments (several thousands of base pairs at 0 mins to $\sim 50-120 \mathrm{bp}$ after 10 mins). DNA prepared with a 10 minute fragmentation time caused the largest increase in EIS signal (Figure 3E). In a previous study, we showed that fragmenting MRSA gDNA for five minutes resulted in successful detection of MRSA by EIS ${ }^{3}$. Here we demonstrate how fragmentation progresses over time and that increased fragmentation causes increased EIS signal over a 10 minute period. In this study, longer fragmentation times than ten minutes were not investigated because longer times would have an adverse effect on the assay time to result. 

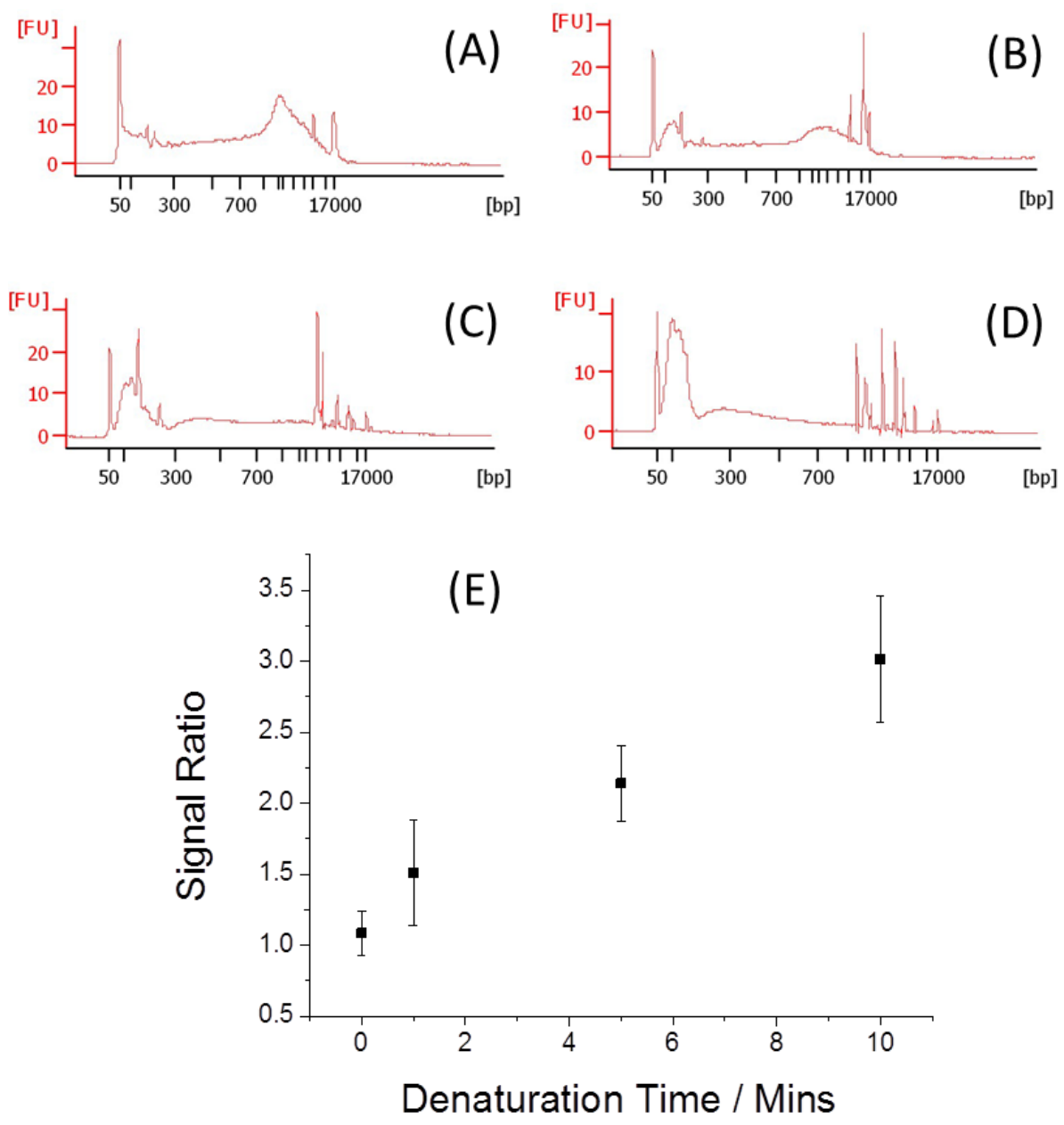

Figure 2. Electropherograms showing DNA fragmentation following incubation at $95^{\circ} \mathrm{C}$ for (A) $0 \mathrm{~min}$ (B) $1 \mathrm{~min}$ (C) $5 \mathrm{~min}$ (D) $10 \mathrm{mins}$. (E) EIS signal ratio responses in batch mode after incubating probe 2 modified electrodes with DNA fragmented for $0,1,5$ and 10 mins.

The findings with clinical samples correlate with those reported from the artificial oligonucleotide sequences in that very long fragments did not boost the EIS signal as might have been expected. For the samples of bacterial gDNA it was shown that heating for up to 10 minutes improved the EIS signal and this was due to the creation of shorter fragments with shorter solution side overhangs. A maximum heat fragmentation of 10 minutes was chosen so as to simulate a satisfactory time to result for a point of care test ${ }^{3}$. It could be that longer fragmentations further improve the signal but it can also be presumed that prolonged fragmentation will eventually reduce the EIS signal due to shortening of the overhangs and destruction of the recognition sequence.

\section{Conclusions}

When binding an artificial target, EIS signal increases were greatest for the $30 \mathrm{nt}$ (15 nt overhang) target sequence. A minor difference in the pattern of signal increases was observed between the batch and kinetic assays. In the batch assay the 60 mer caused the second largest signal increase whereas in kinetic mode the second largest signal increase was brought about by the 15 mer. This finding is 
explained by the different ionic strengths of the solutions involved in the two assays. The information gained by using short artificial targets provides an improved understanding of the nature of the signal increases brought about by solution side overhangs. When binding a real world sample in the form of MRSA genomic DNA a correlation was seen between fragmentation time and EIS signal increases, i.e. comparatively shorter fragments of DNA caused a larger signal increase in the EIS signal. The progressive fragmentation of gDNA and the resultant increase in EIS signal has not been previously shown. This improved understanding of the probe target interaction will inform design of future electrochemical DNA biosensors and allow improved sensitivity to be achieved.

\section{Acknowledgements}

The authors would like to thank Alan Ross, Alison Hardie and Dr Kate Templeton for assistance with MRSA gDNA preparation, molecular biology and capillary gel electrophoresis and Professor Mark Bradley for use of equipment in Chemistry.

\section{Reference List}

(1) Lisdat, F.; Schafer, D. Anal.Bioanal.Chem. 2008.

(2) Bonanni, A.; Esplandiu, M. J.; Pividori, M. I.; Alegret, S.; Valle, M. Analytical and Bioanalytical Chemistry 2006, 385, 1195-1201.

(3) Corrigan, D. K.; Schulze, H.; Henihan, G.; Hardie, A.; Ciani, I.; Giraud, G.; Terry, J. G.; Walton, A. J.; Pethig, R.; Ghazal, P.; Crain, J.; Campbell, C. J.; Templeton, K. E.; Mount, A. R.; Bachmann, T. T. Analyst 2013, 138, 6997-7005.

(4) Bonanni, A.; Esplandiu, M. J.; del Valle, M. Electrochimica Acta 2008, 53, 4022-4029.

(5) Kafka, J.; Panke, O.; Abendroth, B.; Lisdat, F. Electrochimica Acta 2008, 53, 7467-7474.

(6) Keighley, S. D.; Li, P.; Estrela, P.; Mighorato, P. Biosensors \& Bioelectronics 2008, 23, 1291 1297.

(7) Li, D. L.; Zou, X. Q.; Shen, Q.; Dong, S. J. Electrochemistry Communications 2007, 9, 191-196.

(8) Park, J. Y.; Lee, Y. S.; Chang, B. Y.; Kim, B. H.; Jeon, S.; Park, S. M. Anal.Chem. 2010, 82, 83428348.

(9) Patel, M. K.; Solanki, P. R.; Seth, S.; Gupta, S.; Khare, S.; Kumar, A.; Malhotra, B. D. Electrochemistry Communications 2009, 11, 969-973.

(10) Patel, M. K.; Solanki, P. R.; Kumar, A.; Khare, S.; Gupta, S.; Malhotra, B. D. Biosensors \& Bioelectronics 2010, 25, 2586-2591.

(11) Zhou, N.; Yang, T.; Jiang, C.; Du, M.; Jiao, K. Talanta 2009, 77, 1021-1026.

(12) Zhu, N.; Gao, H.; Gu, Y. F.; Xu, Q.; He, P. G.; Fang, Y. Z. Analyst 2009, 134, 860-866. 
(13) Zhu, N.; Gao, H.; Xu, Q.; Lin, Y.; Su, L.; Mao, L. Biosensors and Bioelectronics 2010, 25, 1498 1503.

(14) Ciani, I.; Schulze, H.; Corrigan, D. K.; Henihan, G.; Giraud, G.; Terry, J. G.; Walton, A. J.; Pethig, R.; Ghazal, P.; Crain, J.; Campbell, C. J.; Bachmann, T. T.; Mount, A. R. Biosens.Bioelectron. 2012, 31, 413-418.

(15) Corrigan, D. K.; Schulze, H.; Henihan, G.; Ciani, I.; Giraud, G.; Terry, J. G.; Walton, A. J.; Pethig, R.; Ghazal, P.; Crain, J.; Campbell, C. J.; Mount, A. R.; Bachmann, T. T. Biosensors and Bioelectronics 2012, 34, 178-184.

(16) Degefa, T. H.; Kwak, J. Journal of Electroanalytical Chemistry 2008, 612, 37-41.

(17) Keighley, S. D.; Estrela, P.; Li, P.; Mighorato, P. Biosensors \& Bioelectronics 2008, 24, 906-911.

(18) Tercero, N.; Wang, K.; Gong, P.; Levicky, R. J.Am.Chem.Soc. 2009, 131, 4953-4961.

(19) Riedel, M.; Kartchemnik, J.; Sch+Âning, M. J.; Lisdat, F. Anal.Chem. 2014, 86, 7867-7874.

(20) Johnson, R. P.; Richardson, J. A.; Brown, T.; Bartlett, P. N. J.Am.Chem.Soc. 2012, 134, 1409914107.

(21) Bard A.J.; Faulkner L.R. Electrochemical Methods - Fundamentals and Applications; John Wiley and Sons: 2001.

(22) Ghindilis, A. L.; Smith, M. W.; Schwarzkopf, K. R.; Zhan, C. Q.; Evans, D. R.; Baptista, A. M.; Simon, H. M. Electroanalysis 2009, 12, 1459-1468. 